\title{
Lumbalgia ocupacional en médicos residentes del Hospital Ángeles Mocel
}

\section{Occupational low back pain in resident physicians at Hospital Ángeles Mocel}

\author{
Minseung Jeong, * Rubén García Linage, * Eric Misael Saucedo Moreno, ${ }^{\neq}$ \\ José Fernando Ramos Morales, §imena Alva Rojas"
}

Citar como: Jeong M, García LR, Saucedo MEM, Ramos MJF, Alva RX. Lumbalgia ocupacional en médicos residentes del Hospital Ángeles Mocel. Acta Med Grupo Angeles. 2021; 19 (2): 186-189. https://dx.doi.org/10.35366/100440

\section{Resumen}

La lumbalgia es un término médico utilizado para describir dolor, tensión muscular y/o rigidez en la región lumbar. En México se ha observado que el $13 \%$ de la población (edad de 20 a 59 años) que acude a las Unidades de Medicina Familiar del IMSS manifiesta este tipo de dolor. La lumbalgia ocupacional se caracteriza por dolor de intensidad y duración variable que puede causar incapacidad e invalidez laboral. La prevalencia anual de lumbalgia en personal de salud es aproximadamente de $77 \%$. Se realizó un estudio prospectivo, observacional, transversal. En el cual evaluamos la lumbalgia existente entre los residentes de la rama quirúrgica (Ortopedia y Traumatología, Cirugía General) y la rama no quirúrgica (Medicina Interna). A todos los participantes se les aplicó el test de Oswestry y Roland Morris al inicio del ciclo académico con la intención de reconocer el grado de lumbalgia que presentaban y se volvió a aplicar el mismo test un año después. Los residentes de la rama quirúrgica presentan aumento en lumbalgia a comparación de los residentes no quirúrgicos con una $p=0.007$. Se recomienda que en un futuro se continúe con esta línea de investigación haciendo énfasis en los factores de riesgo que hay entre los residentes quirúrgicos.

Palabras clave: Lumbalgia, lumbalgia ocupacional, médicos residentes.

\footnotetext{
* Médico residente de cuarto año de Ortopedia y Traumatología, Hospital Ángeles Mocel. "Facultad Mexicana de Medicina. Universidad La Salle México".

* Cirugía General y Asesor estadístico, Hospital Ángeles Mocel.

$\S$ Profesor adjunto, Hospital Ángeles Mocel.

" Médico pasante de Servicio Social, Universidad Anáhuac.
}

Correspondencia:

Minseung Jeong

Correo electrónico: min.md@hotmail.com

Aceptado: 04-06-2020.

www.medigraphic.com/actamedica
Abstract

Low back pain is a medical term used to describe pain, muscle tension and/or stiffness in the lower back region. In Mexico, it has been observed that $13 \%$ of the population (age 20 to 59) that attends the Family Medicine Units of the IMSS manifests this type of pain. Occupational low back pain is characterized by pain of variable intensity and duration that can cause disability and occupational invalidity. The annual prevalence of low back pain among health personnel is approximately $77 \%$. We conducted a prospective, observational, cross-sectional study in which we evaluated low back pain among residents of the surgical branch (orthopedics and traumatology, general surgery) and the non-surgical branch (internal medicine). The Oswestry and Roland Morris Test was applied to all participants at the beginning of the academic cycle with the intention of recognizing the degree of low back pain they presented, and the same test was applied after a year later. The residents in the surgical branch present an increase in low back pain compared to non-surgical residents with a $p=0.007$. It is recommended that this line of research be continued in the future, emphasizing the risk factors among surgical residents.

Keywords: Back pain, occupational back pain, medical residents.

\section{INTRODUCCIÓN}

La lumbalgia es un término médico utilizado para describir dolor, tensión muscular y/o rigidez en la región lumbar. ${ }^{1}$ La lumbalgia abarca desde el borde inferior de la última costilla hasta la región del glúteo inferior arriba de las líneas glúteas inferiores con o sin dolor en la parte inferior de las extremidades. ${ }^{2}$

Este tema es de suma relevancia social y económica, ya que es extremadamente común; se estima que $80 \%$ de la población en EUA cursa con al menos un cuadro de lumbalgia a lo largo de su vida. Además de ser una de 
las principales causas de incapacidad y la quinta causa de atención médica. ${ }^{3}$

En México se ha observado que el 13\% de la población (edad de 20 a 59 años) que acude a las Unidades de Medicina Familiar del Instituto Mexicano del Seguro Social (IMSS) manifiesta este tipo de dolor. Se ve afectada por esta patología un cuarto de la población en general, con una estimación de 28 millones de personas en México. ${ }^{4}$

Al ser un término tan basto cuenta con múltiples clasificaciones, las cuales se encargan de englobar una lumbalgia por sus características generales a particulares. Dos grandes grupos en los que se clasifican es dependiendo de si el dolor es específico o inespecífico (80\% de los casos). La lumbalgia inespecífica se refiere a la condición que no se puede atribuir a ninguna otra patología. Mientras que la lumbalgia específica es aquella en la cual se atribuye el dolor a una patología subyacente. ${ }^{5}$ Otra clasificación depende de la temporalidad del dolor, clasificando de esta manera a la lumbalgia en: aguda (dolor lumbar de menos de seis semanas de duración), subaguda (dolor lumbar entre seis a 12 semanas) y crónica (dolor lumbar por más de 12 semanas).

La lumbalgia ocupacional se caracteriza por dolor de intensidad y duración variable que puede causar incapacidad e invalidez laboral. ${ }^{6}$ La lumbalgia es una de las quejas más comunes en personal de salud. La prevalencia anual de lumbalgia en personal de salud es de aproximadamente $77 \%$. Las condiciones de trabajo de los médicos se han considerado como el mayor riesgo para desarrollar trastornos musculoesqueléticos. Los factores de riesgo pueden variar según la especialidad de los médicos; sin embargo, incluyen: bipedestación o sedestación prolongada, mala postura, cargas pesadas, empujar o jalar objetos incluyendo pacientes, movimientos de flexión y rotación. ${ }^{7}$ También falta de ejercicio y factores psicosociales como altos niveles de estrés y trabajos de larga duración, como guardias en los hospitales, se consideran factores de riesgo. ${ }^{8}$

El propósito de este estudio es determinar la prevalencia de lumbalgia en médicos residentes con especialidad de Ortopedia y Traumatología, y Cirugía General, en comparación a la rama no quirúrgica, Medicina Interna, del Hospital Ángeles Mocel durante un año.

\section{MATERIAL Y MÉTODOS}

Se realizó un estudio prospectivo, observacional, transversal, en el cual se evaluó la lumbalgia que existe entre los residentes de la rama quirúrgica, específicamente Ortopedia y Cirugía General, y la rama no quirúrgica (Medicina Interna) en un Hospital de Tercer Nivel de la Ciudad de México en el sector privado.

Se elaboraron dos grupos de estudio: el grupo 1 representa al grupo evaluado y hace referencia a residentes de

\begin{tabular}{lc}
\multicolumn{2}{c}{ Tabla 1: Características de los participantes. } \\
\hline Variables & $\mathrm{n}(\%)$ \\
\hline Masculino & $8(36.4)$ \\
Puesto & \\
R1 & $7(35.0)$ \\
R2 & $7(35.0)$ \\
R3 & $5(25.0)$ \\
R4 & $1(5.0)$ \\
Especialidad & \\
Ortopedia y Traumatología & $7(31.8)$ \\
Cirugía General & $3(13.6)$ \\
Medicina Interna & $12(54.6)$ \\
Tabaquismo & $5(22.7)$ \\
Actividad física & $13(59.1)$ \\
&
\end{tabular}

la rama quirúrgica (Traumatología y Ortopedia); el grupo 2 es el grupo control y representa a residentes de la rama no quirúrgica (Medicina Interna). A todos los participantes se les aplicó el test de Oswestry y Roland Morris al inicio del ciclo académico, con la intención de reconocer de manera objetiva el grado de lumbalgia que presentaban y se volvió aplicar el mismo test un año después.

Se utilizó la prueba de Wilcoxon para datos pareados para comparar los resultados. El análisis estadístico se realizó con el software SPSS versión 20.0 (SPSS, Chicago, EUA). Se realizó un análisis de Kolmogorov-Smirnov para determinar la distribución de las variables cuantitativas, y con base en dicha distribución se resumieron mediante media y desviación estándar o mediana, mínimo y máximo según el caso. Aquellos con distribuciones no paramétricas fueron analizados con la prueba $U$ de Mann-Whitney y prueba exacta de Fisher. Se consideró un valor significativo de $p=0.05$ y un intervalo de confianza del $95 \%$.

\section{RESULTADOS}

Se evaluó una muestra total de 22 participantes. Se excluyeron a los residentes que no pudieron contestar el test en el siguiente año porque se derivaron a otras especialidades o se encontraban fuera del hospital. Se clasificaron de acuerdo con el cargo que tienen dentro del hospital: siete residentes del Servicio de Ortopedia, tres residentes de Cirugía General y 12 residentes de Medicina Interna. De todos los participantes ocho representan al sexo masculino (36.4\%) y el restante son femeninos (Tabla 1).

La muestra es pequeña debido a que fue realizado en un hospital privado donde cuenta con sólo servicio de Ortopedia y Traumatología, Cirugía General, Medicina Interna, Imagenología y Terapia Intensiva. 
Se dividió la muestra en dos grupos: el grupo 1 con residentes de la rama quirúrgica y el grupo dos con residentes de la rama no quirúrgica. Al comparar los grupos se identificaron diferencias significativas para la talla $(p=$ 0.063 IC95\% -0.005 a 0.150), peso ( $p=0.004$ IC95\% 6.16 a 26.11), IMC ( $p=0.005$ IC 95\% 1.23 a 6.00) (Tabla 2).

Al realizar la prueba de Wilcoxon para datos pareados, en los que se compararon los resultados de las pruebas de Oswestry y Roland Morris del año 2018 contra los resultados de 2019, se identificó que para el grupo del residente de la rama quirúrgica existe aumento de los valores del test Oswestry ( $p=0.007$ ) en comparación con el grupo 2, en el cual los valores se mantuvieron en un rango muy similar $(p=0.779)$. Al comparar la prueba de Roland Morris no se identificaron diferencias significativas para ambos grupos ( $p=0.67$ y $p=0.290$, respectivamente), aunque se observa una tendencia en obtener valores más elevados para el grupo de residentes de la rama quirúrgica (Tabla 3).

\section{DISCUSIÓN}

Almalki y colaboradores, en su estudio en el que analizaron la prevalencia y los efectos de la lumbalgia en los profesionales de la salud en King Abdulaziz Medical City (KAMC) en Riyadh, reportaron que los factores de riesgo de presentar la lumbalgia son: edad, sexo femenino, alto IMC, falta de ejercicio y trabajar más de cuatro horas en sedestación o bipedestación. ${ }^{9}$ Landry y su equipo realizaron una encuesta transversal de 344 pacientes, en el cual observaron que los siguientes factores se asociaron con lumbalgia: contacto directo con los pacientes $(p=0.015)$, levantamiento $y$ posicionamiento de los pacientes $(p=0.016)$ y baja satisfacción laboral $(p=0.039)$. La proporción de prevalencia reportó que la lumbalgia aguda fue directamente proporcional a la cantidad de levantamientos y posicionamiento de los pacientes que se realiza al día (prevalence ratio [PR: 1.4; $95 \% \mathrm{Cl}: 1.27$ a 1.7]). ${ }^{10}$ En comparación con nuestro estudio podemos observar que el grupo quirúrgico tiene mayor prevalencia de lumbalgia debido a los factores de riesgo previamente comentados.

Bejia y sus colegas, en su estudio en el que realizaron un cuestionario a 350 empleados del Hospital Fattouma Bourguiba, reportaron que los factores de riesgo asociados a la lumbalgia son la edad ( $p \leq 0.01)$, alto IMC ( $p=$ $0.01)$ y levantamiento de objetos pesados $(p=0.008)$. Se observó que el ejercicio es un factor protector $(p=$ 0.019). ${ }^{11}$ Wong y su grupo, en su encuesta transversal de 931 participantes, concluyeron que los factores de riesgo asociados a la lumbalgia fueron: mala postura $(p=0.001)$, levantamiento de objetos pesados o pacientes $(p \leq 0.001)$, nivel de satisfacción del trabajo $(p \leq 0.001)$ y niveles de estrés $(p=0.001) .{ }^{12}$ En nuestro estudio no se analizaron factores como nivel de satisfacción del trabajo ni el nivel de estrés; sin embargo, se observó que los residentes de la rama quirúrgica tenían mayor prevalencia de levantamiento de objetos pesados como pacientes, mayor IMC (26.18 vs 21.35 ) y menor porcentaje de actividad física (20 vs $58.3 \%$ ).

En el cuestionario de este artículo no se mencionaron variables como el posicionamiento erróneo, el levantamiento y el posicionamiento de los pacientes. Pero es necesario tomar en cuenta que los residentes de la rama quirúrgica tienen mayor exposición a cualquiera de estas variables y factores de riesgo.

Es importante mencionar que en los estudios aquí presentados, al igual que muchos otros, tienen como factor de riesgo independiente para lumbalgia ocupacional la presencia de peso e IMC elevados, siendo congruentes los

\begin{tabular}{|c|c|c|c|c|}
\hline & Quirúrgico & No quirúrgico & $p^{\ddagger}$ & IC $95 \%$ \\
\hline Edad & $29(8-25)$ & $26(22-36)$ & 0.124 & -0.625 a 4.791 \\
\hline Masculino, n (\%) & $6(60.0)$ & $2(16.7)$ & 0.074 & - \\
\hline Talla* & $1.76(1.6-1.7)$ & $1.64(1.6-1.7)$ & 0.063 & -0.005 a 0.150 \\
\hline Peso* & $78.5(51.5-96)$ & $56(50-75)$ & 0.004 & 6.16 a 26.110 \\
\hline Índice de masa corporal* & $26.18(20.6-29.6)$ & $21.35(18.4-26.0)$ & 0.005 & 1.23 a 6.000 \\
\hline Sueño en guardias* & $3(2-3)$ & $2.5(1-4)$ & 0.502 & -0.273 a 0.540 \\
\hline Guardias a la semana* & $3(2-3)$ & $3(2-3)$ & 1.000 & -0.376 a 0.976 \\
\hline Actividad física, n (\%) & $2(20.0)$ & 7 (58.3) & 0.099 & - \\
\hline Tabaquismo, n (\%) & $2(20.0)$ & $3(25.0)$ & 1.000 & - \\
\hline $\begin{array}{l}\text { * Media (rango). } \\
\text { ₹ Valor significativo de } p=0.0 \\
\text { IC } 95 \%=\text { intervalo de confiar }\end{array}$ & & & & \\
\hline
\end{tabular}


Tabla 3: Comparación de los resultados de las pruebas del Oswestry y Roland Morris.

\begin{tabular}{|c|c|c|c|}
\hline & 2018 & 2019 & $\mathrm{p}$ \\
\hline \multicolumn{4}{|c|}{ Oswestry (grupo) } \\
\hline 1 & $1(0-38)$ & $20(0-80)$ & 0.007 \\
\hline 2 & $21(0-32)$ & $22(0-38)$ & 0.779 \\
\hline \multicolumn{4}{|c|}{ Roland Morris (grupo) } \\
\hline 1 & $1.5(0-8)$ & $2(0-24)$ & 0.671 \\
\hline 2 & $1(0-3)$ & $1(0-3)$ & 0.290 \\
\hline
\end{tabular}

resultados encontrados en nuestro estudio donde fueron estas dos variables las únicas con significancia estadística en la comparación de proporciones con una $\mathrm{p}=0.004$ (IC95\% 6.16-26.11) у p $=0.005$ (IC95\% 1.23-6.00), respectivamente. Esto podría crear un sesgo en los resultados, siendo completamente dependientes del peso y no sólo de las actividades y ocupación que realizan los residentes de las ramas quirúrgicas frente a los no quirúrgicos. Sin embargo, es un sesgo que no podemos eliminar, puesto que el número de residentes es pequeño en nuestra población hospitalaria para poder sólo incluir residentes sin sobrepeso.

Creemos que esta variabilidad en el peso entre residentes de ambas ramas es debido, probablemente, a pasar mayor tiempo dentro del quirófano y/o áreas estériles en las que no es permitido tener acceso a alimentos y el horario de trabajo puede no estar tan calendarizado u organizado como en áreas no quirúrgicas, en las que es más fácil una organización de tiempos y de igual forma mejores hábitos alimenticios y actividad física. Por esta razón, se deberán hacer estudios prospectivos en los cuales se tomen en cuenta estas variables y poder definir si aportan significancia o son una hipótesis.

La lumbalgia ocupacional en el caso de los residentes de la rama quirúrgica aumentó significativamente en un año de residencia; mientras que los residentes de áreas no quirúrgicas no muestran un incremento de lumbalgia significativa durante este año.

Este estudio tiene un valor clínico significativo, ya que, aunque anteriormente se han hecho múltiples artículos sobre la lumbalgia ocupacional en los profesionales de la salud, los artículos que hacen una distinción entre residentes en área quirúrgica vs residentes no quirúrgicos son limitados.

Los residentes de la rama quirúrgica se enfrentan a un número exponencial de factores de riesgo; entre ellos largas horas de posiciones incorrectas (por las malas posturas adquiridas en diferentes cirugías), además del levantamiento y posicionamiento de pacientes en el quirófano.
Por otro lado, este artículo cuenta con limitantes, ya que se necesita un mayor número de participantes para que la muestra estudiada sea un verdadero reflejo del total de la población estudiada.

\section{CONCLUSIÓN}

Se corroboró que los residentes en la rama quirúrgica presentan un aumento en la lumbalgia comparados con los residentes no quirúrgicos con una $\mathrm{p}=0.007$. Por los resultados de este estudio se recomienda que en un futuro se continúe con esta línea de investigación, haciendo énfasis en los factores de riesgo que hay entre los residentes quirúrgicos. De esta manera, en un futuro se podrán reducir los casos de lumbalgia al eliminar o controlar los factores de riesgo a los cuales se enfrentan los profesionales de la salud en áreas quirúrgicas.

\section{REFERENCIAS}

1. Airaksinen O, Brox JI, Cedraschi C, Hildebrandt J, Klaber-Moffett J, Kovacs $\mathrm{F}$ et al. Chapter 4 European guidelines for the management of chronic nonspecific low back pain. Eur Spine J. 2006; 15 Suppl 2 (Suppl 2): S192-300.

2. Chavarría SJ. Lumbalgia: Causas, diagnóstico y manejo. Rev Med Cos Cen. 2014; 71 (611): 447-454.

3. Patrick N, Emanski E, Knaub MA. Acute and chronic low back pain. Med Clin North Am. 2014; 98 (4): 777-789.

4. Covarrubias-Gómez A. Papel del anestesiólogo en el manejo del dolor crónico. Rev Mex Anest. 2008; 31 (Suppl. 1): S235-S237.

5. Thiese MS, Hegmann KT, Garg A, Porucznik C, Behrens T. The predictive relationship of physical activity on the incidence of low back pain in an occupational cohort. J Occup Environ Med. 2011; 53 (4): 364-371.

6. Helfenstein Junior M, Goldenfum MA, Siena C. Occupational low back pain. Rev Assoc Med Bras. 2010; 56 (5): 583-589.

7. Shams Vahdati S, Sarkhosh Khiavi R, Rajaei Ghafouri R, Adimi I. Evaluation of prevalence of low back pain among residents of tabriz university of medical sciences in relation with their position in work. Turk J Emerg Med. 2014; 14 (3): 125-159.

8. Al-Mendalawi M. Prevalence of low back pain among medical practitioners in a tertiary care hospital in Riyadh. Saudi Journal of Sports Medicine. 2016; 16 (3): 205-209.

9. Almalki M, Alkhudhayri M, Batarfi A, Alrumaihi S, Alshehri S, Alkenani $\mathrm{N}$ et al. Prevalence of low back pain among medical practitioners in a tertiary care hospital in Riyadh. Saudi Journal of Sports Medicine. 2016; 16 (3): 205-029.

10. Landry MD, Raman SR, Sulway C, Golightly YM, Hamdan E. Prevalence and risk factors associated with low back pain among health care providers in a Kuwait Hospital. Spine. 2008; 33 (5): 539-545.

11. Bejia I, Younes M, Jamilia HB, Khalfallah T, Ben Salem K, Touzi M et al. Prevalence and risk factors associated to low back pain among hospital staff. Joint Bone Spine. 2005; 72 (3): 253-259.

12. Wong T, Teo N, Kyaw M. Prevalence and risk factors associated with low back pain among health care providers in a district hospital. Malaysian Orthopaedic Journal. 2010; 4 (2): 23-28. 